\title{
GWAS of brain volume on 54,407 individuals and cross-trait analysis with intelligence identifies shared genomic loci and genes
}

Philip R Jansen ${ }^{1,2,3 \dagger}$, Mats Nagel ${ }^{1,2 \dagger}$, Kyoko Watanabe ${ }^{1}$, Yongbin Wei $^{4}$, Jeanne E Savage ${ }^{1}$, Christiaan A de Leeuw ${ }^{1}$, Martijn P van den Heuvel2,4, Sophie van der Sluis ${ }^{1,2 \S}$, Danielle

$$
\text { Posthuma }{ }^{1,2 \S^{*}}
$$

\section{Affiliations:}

1 Department of Complex Trait Genetics, Center for Neurogenomics and Cognitive Research, Amsterdam Neuroscience, Vrije Universiteit, Amsterdam, The Netherlands

2 Department of Clinical Genetics, Section Complex Trait Genetics, Amsterdam Neuroscience, Vrije Universiteit Medical Center, Amsterdam UMC, Amsterdam, the Netherlands

3 Department of Child and Adolescent Psychiatry, Erasmus University Medical Center, Rotterdam, the Netherlands

4 Connectome Group, Department of Complex Trait Genetics, Center for Neurogenomics and Cognitive Research, Amsterdam Neuroscience, Vrije Universiteit, Amsterdam, the Netherlands

† These authors contributed equally to this work

$\S$ These authors jointly supervised this work

${ }^{*}$ Correspondence should be addressed to: Danielle Posthuma: Department of Complex Trait Genetics, Vrije Universiteit Amsterdam, De Boelelaan 1085, 1081 HV, Amsterdam, The Netherlands. Phone: +31 20598 2823, Fax: +31 20 5986926, d.posthuma@vu.nl 


\section{Abstract}

2 The phenotypic correlation between human intelligence and brain volume (BV) is considerable

$3(r \approx 0.40)$, and has been shown to be due to shared genetic factors ${ }^{1}$. To further examine specific

4 genetic factors driving this correlation, we present genomic analyses of the genetic overlap

5 between intelligence and BV using genome-wide association study (GWAS) results. First, we

6 conducted the largest BV GWAS meta-analysis to date ( $N=54,407$ individuals), followed by

7 functional annotation and gene-mapping. We identified 35 genomic loci (27 novel), implicating

8362 genes (346 novel) and 23 biological pathways for BV. Second, we used an existing GWAS for

9 intelligence $\left(\mathrm{N}=269,867\right.$ individuals $\left.^{2}\right)$, and estimated the genetic correlation $\left(r_{g}\right)$ between BV

10 and intelligence to be 0.23 . We show that the $r_{g}$ is driven by physical overlap of GWAS hits in 5

11 genomic loci. We identified 67 shared genes between BV and intelligence, which are mainly

12 involved in important signaling pathways regulating cell growth. Out of these 67 we prioritized

1332 that are most likely to have functional impact. These results provide new information on the

14 genetics of BV and provide biological insight into BV's shared genetic etiology with intelligence.

15

16 
GWAS of brain volume

18 To identify SNPs associated with brain volume (BV), we first performed a GWAS

19 (Supplementary Fig. 1) using data of 17,062 participants from the UK Biobank ${ }^{3}$, with BV

20 estimated from structural ( $\mathrm{T}_{1}$-weighted) magnetic resonance imaging (MRI) by summing total

21 gray and white matter volume, and ventricular cerebrospinal fluid volume (Supplementary

22 Fig. 2). These results were then meta-analyzed with GWAS results from two previously

23 published studies ${ }^{4,5}$ : one on intracranial volume (ICV) from the ENIGMA-CHARGE ${ }^{4}$ collaboration

24 ( $N=21,875$ individuals), and a second one on head circumference, a proven proxy of BV6-8, from

25 the Early Growth Genetics (EGG) consortium ${ }^{5}$ ( $N=10,768$ individuals). This led to a total sample

26 size of 54,407 unrelated Europeans (Supplementary Fig. 3; Supplementary Methods 1.1-1.2;

27 Supplementary Table 1). Linkage disequilibrium score regression (LDSC; ${ }^{9}$ Online Methods)

28 showed high concordance of SNP associations between the three samples (UKB and ENIGMA-

29 CHARGE: $r_{g}=1.04$, SE=0.07; UKB and EGG: $r_{g}=0.80$, SE=0.14; ENIGMA-CHARGE \& EGG: $r_{g}=0.71$,

30 SE=0.15; Supplementary Table 2), justifying subsequent meta-analysis. Sample-size weighted

31 fixed-effects meta-analysis was carried out using METAL ${ }^{10}$ (Online Methods) resulting in 46

32 linkage disequilibrium (LD) independent lead SNPs $\left(r^{2}<0.1\right)$, residing in 35 genomic loci (Fig.

33 1a; Supplementary Table 3; Supplementary Fig. 4), representing 4,583 genome-wide

34 significant SNPs (Fig. 1b, Supplementary Table 4) associated with our measure of BV. Of these

3535 loci, 27 were novel compared to the latest GWAS study of intracranial volume 4 . To see if our

36 results were driven by only one of the three samples or were supported by all three, we

37 examined the direction of effect of all genome-wide significant (GWS) SNPs across the three

38 individual cohorts. We found high concordance: $99.5 \%$ of the 4,583 SNPs had the same

39 direction of effect in all three cohorts. Of the 4,583 SNPs that were GWS in the BV meta-analysis,

$4015 \%$ was GWS in at least two of the individual cohorts, while $77 \%(19.7 \%)$ had a $P$-value $<0.05$

41 in at least two (all three) cohorts. The SNP-heritability $\left(h^{2}{ }_{S N P}\right)$ of BV estimated by LDSC was 0.24

42 ( $S E=0.02)$, and the LDSC intercept approximated $1(1.037, S E=0.009)$, suggesting that the

43 inflation in test statistics (Lambda $\lambda_{\mathrm{GC}}=1.23$ ) was largely due to polygenicity and not to 
44 unaccounted for population stratification ${ }^{11}$. Functional annotation of 5,802 'candidate' SNPs

45 (i.e., SNPs in the risk loci with a GWAS $P$-value of $P<10^{-5}$ and LD $r^{2}>0.6$ with one of the

46 independent significant SNPs; Online Methods) carried out in FUMA ${ }^{12}$ showed that these SNPs

47 were most abundant in intronic $(n=2,879,49.6 \%)$ or intergenic regions $(n=1,131,19.5 \%)$, these

48 proportions were significantly enriched (compared to $37.5 \%, P=1.63 \times 10^{-81}$ ) or depleted

49 (compared to $45.6 \%, P<1 \times 10^{-100}$ ) relative to all SNPs included in the meta-analysis, respectively

50 (Supplementary Fig. 5; Supplementary Table 5), and 30 (0.5\%) were exonic

51 nonsynonymous SNPs (ExNS) altering protein structures of 15 genes (Supplementary Table 6,

52 Supplementary Results 2.1). One gene, SPPL2C, contained 8 ExNS (all in exon 1, and in the

53 same inversion region). SPPL2C codes for the signal peptide peptidase-like $2 \mathrm{C}$, which plays a

54 role in the degradation of signaling peptides in the brain ${ }^{13}$.

55 To test whether specific functional categories SNP annotations contribute disproportionally to

56 the heritability of BV, we used LDSC to partition the genetic signal over functional SNP

57 categories (Online Methods). We observed significant heritability enrichment in 13 SNP

58 categories (Fig. 1c; Supplementary Table 7), with the strongest enrichment of SNPs in

59 (evolutionary) conserved regions, (enrichment=12.8, $\mathrm{SE}=2.3, P=1.03 \times 10^{-6}$, suggesting an 12.8 -

60 fold increase in $h^{2}$ conveyed by SNPs in these regions), H3K9ac peaks (i.e., specific histone

61 modification that is correlated with active promoters; enrichment $=10.1, \mathrm{SE}=2.5, P=5.58 \times 10^{-4}$ )

62 and transcription start sites (TSS; enrichment=9.5, SE=2.5, $P=8.48 \times 10^{-4}$ ).

\section{$64 \quad$ BV gene analyses}

65 To gain insight into which genes may be involved in BV, we mapped the candidate SNPs

66 implicated in the BV meta-analysis to genes, using positional mapping, eQTL mapping, and

67 chromatin interaction mapping as implemented in FUMA ${ }^{12}$, and by performing gene-based association tests as implemented in MAGMA $^{14}$ (Online Methods, Supplementary Fig. 6). In total, 362 unique genes were implicated by at least one of these methods (of these, 140 genes were overlapping with one of the 35 identified risk loci). Specifically, the 35 risk loci were 
71 mapped to 101 genes based on position, 186 genes by eQTL association, 201 genes through

72 chromatin-chromatin interactions (Fig. 2a; Supplementary Table 8), and gene-based

73 association testing identified 70 genes (Fig. 2b; Supplementary Table 9). The 362 genes

74 included 17 genes that were replicated from previous studies (5 not replicated), while 345 were

75 novel gene findings (Supplementary Table 10). Overall, 16 genes were implicated by all

76 methods (CDK6, PRMT5, ERBB3, FOXO3, FRZB, FAM49B, PRR13, WBP1L, HAUS4, RBM23, PTEN,

77 RAB5B, MAP3K12, HMGA2, AJUBA, INA, of which 13 novel gene findings).

78 Since monogenic Mendelian disorders are often characterized by abnormal brain development,

79 we performed look-up of the 362 genes implicated in the BV GWAS in the Online Mendelian

80 Inheritance in Man database ${ }^{15}$ (OMIM, URLs). We identified 106 monogenic disorders caused by

81 high-penetrance mutations in 88 of the 362 genes $(24.3 \%$ vs. $17.1 \%$ in all genes that were

82 tested in the gene-based analysis, $P=3.39 \times 10^{-4}$ ) implicated in BV, many of which are commonly

83 associated with abnormal brain development (including micro- (CDK6) and macrocephalia

84 (PTEN)), and abnormal growth (including leprechaunism (INSR) and tetra-amelia syndrome

85 (WNT3)) (Supplementary Table 11).

86 To identify functional pathways related to BV, we performed gene-set analysis in

87 MAGMA ${ }^{16}$ (Online Methods). Of the 7,246 tested gene-sets (including canonical pathways and

88 gene ontology gene-sets), 23 were significantly associated after Bonferroni correction (Fig. 2c;

89 Supplementary Table 12-13). Among these significant gene-sets were several cell-signaling

90 pathways, including the ErBB2/ErBB4 signaling, the GAB1 signaling pathway and the insulin

91 pathway, and developmental processes such as neuron axogenesis and mesenchyme

92 development. That is, key cell-signaling pathways were implicated that are involved in normal

93 brain development ${ }^{17}$ and involved in several brain developmental abnormalities ${ }^{18,19}$. Pairwise

94 conditional gene-set analysis indicated that several gene-sets (e.g. neuron axogenesis and

95 mesenchyme development) constitute independent associations. (Supplementary Fig. 7-9,

96 Supplementary Table 14, Supplementary Results 2.4). Conversely, conditional analyses

97 suggest that several signaling pathways (e.g., PI3K events in ERBB2/ERBB4 signaling, GAB1 
98 signalosome) represent a shared underlying signal, since conditional $P$-values were

99 substantially higher than the unconditional $P$-values.

100 Next we aimed to identify tissue categories and neuronal cell-types that are enriched for gene

101 signal of BV, by linking gene $P$-values to gene-expression in 53 tissue-types ${ }^{20}$ and 565 brain cell-

102 types $^{21}$ (Online Methods). None of the associations of tissue or cell-types passed our stringent

103 multiple testing correction (i.e. correcting for all tested gene-sets, tissues and cell types, thus

$1040.05 / 7,864)$, but the strongest evidence of association was observed for several types of

105 polydendrocytes, including TNR-PDGFA-PIK3R3 $\left(P=7.46 \times 10^{-4}\right)$ and TNR-CSPG5 $\left(P=2.53 \times 10^{-3}\right)$

106 polydendrocytes (Supplementary Table 12). Polydendrocytes are thought to be precursor

107 cells of oligodendrocytes ${ }^{22}$, a cell-type involved in supporting neuronal health and myelinization

108 of the brain ${ }^{23}$.

109 To determine to what extent our BV GWAS results were the product of a heterogeneous

110 phenotype (structural MRI and head circumference), an additional meta-analysis was

111 performed on a stricter, fully height-adjusted, BV phenotype. The outcomes resembled those of

112 the primary meta-analysis very closely $\left(r_{g}=0.96, \mathrm{SE}=0.007\right)$, increasing our confidence in the

113 main results (see Supplementary Tables $\mathbf{1 5 - 2 2}$ and Supplementary Figs. 10-12 for the

114 results of the SNP- and gene-level analysis, which are discussed in Supplementary Results

$115 \quad 2.5)$

116

117 Genetic correlations between BV and other traits

118 Twin studies have shown substantial genetic overlap between BV and behavioral traits,

119 including intelligence ${ }^{1,24-26}$. We used LDSC to estimate the overlap in genetic signal between BV

120 and 25 brain-related and neuropsychiatric traits for which published summary statistics based

121 on large samples were available (Online Methods). Significant genetic correlations were

122 observed between BV and 6 traits: positive genetic correlations with educational attainment

$123\left(r_{g}=0.25, \mathrm{SE}=0.02, P=5.14 \times 10^{-28}\right.$; Fig. 1d; Supplementary Table 23$)$ and intelligence $\left(r_{g}=0.23\right.$,

$\left.124 \mathrm{SE}=0.03, P=2.82 \times 10^{-15}\right)$, and negative genetic correlations with ADHD $\left(r_{g}=-0,21 \mathrm{SE}=0.04\right.$, 
$\left.125 P=1.97 \times 10^{-7}\right)$, type 2 diabetes $\left(r_{g}=-0.12, \mathrm{SE}=0.04, P=9.56 \times 10^{-4}\right)$, insomnia $\left(r_{g}=-0,13 \mathrm{SE}=0.02\right.$,

$\left.126 P=6.01 \times 10^{-8}\right)$, and neuroticism $\left(r_{g}=-0.13, \mathrm{SE}=0.03, P=1.01 \times 10^{-5}\right)$, confirming previously reported

127 overlap ${ }^{4,27,28}$ and establishing a new association with type 2 diabetes.

129 Genetic overlap with intelligence

130 Development of human intelligence has coincided with a strong increase in total size of the

131 brain ${ }^{29}$. Indeed, epidemiological studies have shown overlap in genetic factors between BV and

132 intelligence ${ }^{1,2}$, which we confirmed here: the estimated $r_{g}$ between BV and intelligence was 0.23

133 (Supplementary Tables 2, 23), closely resembling the estimates of shared genetic factors

134 between intelligence and gray matter volume $\left(r_{g}=0.29\right)$ and white matter volume $\left(r_{g}=0.24\right)$

135 obtained from twin studies ${ }^{1}$. To gain insight into the specific genetic factors driving this genetic

136 correlation, we explored the overlap in genetic signal between the current BV GWAS meta-

137 analysis and a recent large GWAS meta-analysis of intelligence ${ }^{2}(\mathrm{~N}=269,867)$.

138 We first determined whether strongly associated SNPs show the same direction of effect in both

139 traits, by performing a look-up of the lead SNPs of BV in the intelligence GWAS, and vice versa.

140 In line with expectations, we observed a strong sign concordance of the 46 BV lead SNPs in

141 intelligence (sign concordance=89.1\%, $P=3.10 \times 10^{-7}$, Fig. 3c) and weaker but still considerable

142 sign concordance of the 243 lead SNPs of intelligence in BV (sign concordance $=62.1 \%$,

$143 P=1.86 \times 10^{-4}$, Fig. 3d). Similarly, strong deviations from the expected $P$-value distribution were

144 observed for genes that were significant for BV (70) and intelligence (507) in the gene-based

145 test in MAGMA (Supplementary Fig. 13).

146 To explore whether BV and intelligence are causally related, we carried out GWAS summary

147 statistics-based Mendelian Randomization analyses, using the Generalized Summary-data-based

148 Mendelian Randomization package ${ }^{30}$ (GSMR; Online Methods), essentially using independent

149 lead SNPs as instrumental variables (LD: $r^{2}<0.1$ ). GSMR analyses demonstrated a directional

150 effect of BV on intelligence $\left(b_{x y}=0.140, \mathrm{SE}=0.011, P=7.36 \times 10^{-37}\right.$; Fig. 3 a $)$ and a less strong yet still

151 highly significant directional effect of intelligence on $\mathrm{BV}\left(b_{x y}=0.194, \mathrm{SE}=0.025, P=3.58 \times 10^{-15}\right.$; Fig. 
152 3b), suggesting a bidirectional association between these phenotypes, in line with previous

153 reports ${ }^{2}$. We do note, however, that GSMR makes several strong assumptions, such as the

154 absence of a third, mediating, factor, and no horizontal or vertical pleiotropy ${ }^{31}$, which may not

155 always hold, and that the current results should be interpreted conditional on these

156 assumptions.

157 To investigate whether specific SNPs or genes could be identified that drive the genetic overlap

158 between intelligence and BV, we performed several cross-trait analyses of SNPs and genes

159 significantly implicated in both traits. We observed physical overlap in five out of the 35

160 genomic loci for BV, and overlap in genes implicated by FUMA ( $n_{\text {genes }}=45$ ) and MAGMA

161 ( $\left.\mathrm{n}_{\text {genes }}=23\right)$, resulting in 67 unique overlapping genes (Fig. 3e-f; Supplementary Table 24).

162 Conversely, we identified 295 genes for BV that were not significantly associated to intelligence

163 (Fig. 3e). Lookup of gene functions of the 67 overlapping genes in the online GeneCards ${ }^{32}$

164 repository (URLs) showed strong involvement of these genes in a wide variety of cellular

165 processes and key factors in cell division (Supplementary Results 2.6). When comparing the

166 probability of being loss-of-function (LoF) intolerant (pLI score $>0.9$ ) between overlap genes

167 and genes observed for only one of these traits, we observe a slightly higher fraction of genes

168 being LoF intolerant in the genes that play a role in both traits $(32.8 \%$ (shared) vs. $26.0 \%$

169 (intelligence) and 30.2\% (BV), Fig. 3g,). The mutation intolerance of these overlapping genes is

170 further demonstrated by a high fraction of genes in this category that are associated with

171 monogenic disease (23.8\%, Fig. 3g) in the OMIM database), compared to BV-only (18.9\%) and

172 intelligence-only (17.2\%) genes. The finding that the overlapping genes, that are involved in

173 multiple key cellular processes, are overall less tolerant to (LoF) mutations, implies that their

174 unperturbed functioning is crucial to a well-functioning human brain.

175

176 In order to determine whether the set of genes that overlap between BV and intelligence are

177 randomly expressed across cortical areas or show similar expression profiles across cortical

178 brain areas, we examined their cortical gene expression profiles using data obtained from the 
179 Allen Human Brain Atlas ${ }^{33}$ (AHBA). We compared the gene expression profile of the 67 genes

180 related to both $\mathrm{BV}$ and intelligence to that of genes associated to either or both of the traits

181 ( $n_{\text {genes }}=1,852$; Fig. 3e). Specifically, using permutation analysis we compared the expression of

182 these 67 genes across 57 brain regions to that of 10,000 randomly selected, equally large, sets of

183 genes drawn from the 1,852 genes related to either or both of the traits. Although we observed

184 overexpression of the 67 overlapping genes in the anterior part of the fusiform $\left(P=8.12 \times 10^{-3}\right)$

185 and the posterior part of the inferior temporal cortex $\left(P=7.82 \times 10^{-3}\right)$, none of the associations

186 survived a stringent Bonferroni correction $\left(P<8.77 \times 10^{-4}(=0.05 / 57)\right.$; Supplementary Table

187 25). To examine whether clusters of more homogeneously expressed genes exist within the 67

188 overlapping genes, we additionally performed clustering analysis on the correlations between

189 the expression profiles of genes, aiming to maximize intra-cluster cohesion, while at the same

190 time maximizing differences between clusters (see Online Methods). Two clusters were

191 identified (Supplementary Results, Supplementary Table 25) but their expression profiles

192 were not significantly different from those of random sets. Interaction analyses in MAGMA (see

193 Online Methods), testing whether gene expression in specific brain regions contributed

194 significantly to the genetic relationship between BV and intelligence, also turned out negative

195 (Supplementary Table 26). Based on these results, we conclude that the set of overlapping

196 genes do not seem to be expressed in any particular cortical region.

198 To prioritize those genes from the 67 overlapping genes, that are more likely to have causal

199 effects on both BV and intelligence, we filtered the set of common genes based on either one of

200 three conditions: 1) the gene contained an ExNS SNP, 2) the gene was part of one of the

201 significant gene sets for BV or intelligence, and 3) the GWAS signal of either trait colocalized

202 with eQTL signals, using COLOC 34 (colocalization of GWAS and eQTL signals is compatible with

203 the hypothesis of a common causal variant; see URLs; Supplementary Table 24). After filtering

204 the 67 genes that were associated with both BV and intelligence, a selection of 32 potentially

205 functionally interesting genes (17 of which did not physically overlap with each other) 
206 remained. For example, we prioritized two genes on chromosome 3 (out of 6 associated genes

207 in the same region) that may proof particularly interesting candidates for functional follow-up

208 aimed at characterizing the genetic relation between BV and intelligence: USP19 was selected

209 because it contains an ExNS variant that was significantly associated to intelligence, and TFDP2

210 because it was part of a significantly associated gene set for BV.

211 Interestingly, conditional gene-set analyses (where we conditioned gene-based signal in BV on

212 the gene-based Z-scores for intelligence, and vice versa) indicated that the gene sets identified

213 for BV (23) and intelligence (6) are trait specific, since the association remains virtually

214 unchanged when conditioning on the other trait (Supplementary Table 27). Fisher's exact

215 tests showed that the 67 overlapping genes were not significantly enriched in the 29 gene sets

216 associated to BV or intelligence (Supplementary Table 28). However, several genes related to

217 intelligence were located in gene-sets observed for intelligence, including TFDP2, CDKN1A,

218 FOXO3 and VARS1 (see Fig. 4), suggesting that, although no single gene-set was shared between

219 BV and intelligence, several genes associated with intelligence are located along important

220 signaling pathways implicated in BV.

222 In conclusion, we studied the genetics of BV and report 29 novel loci and 346 novel genes for 223 BV. In exploring the genetic overlap between BV and intelligence, we identified 67 genes that 224 are associated to both traits, i.e., contributing to the genetic overlap between these traits. Of the 22567 overlapping genes, we prioritized 32 genes that might be best candidates for functional 226 follow-up aimed at gaining biological insight into the overlap between BV and intelligence. We 227 did not identify specific biological characteristics or mechanisms that differentiate the 67 228 overlapping genes from the full set of genes that was investigated, except for an enriched intolerance to (LoF) mutations. We expect that increasing the sample size, and thus the statistical power, for brain volume GWAS will help in further dissecting the shared nature of

231 brain volume and intelligence. These findings are a first step towards understanding of the 232 genetic architecture of BV and its shared etiology with intelligence. 
a

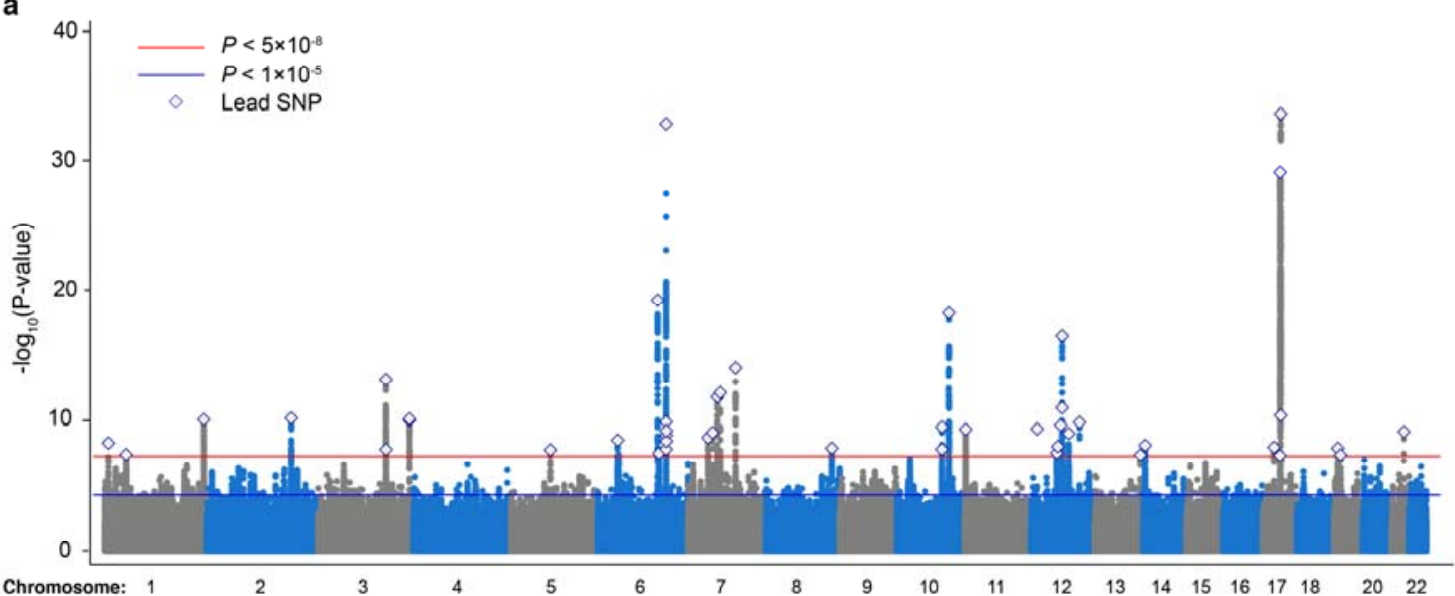

b

c

d

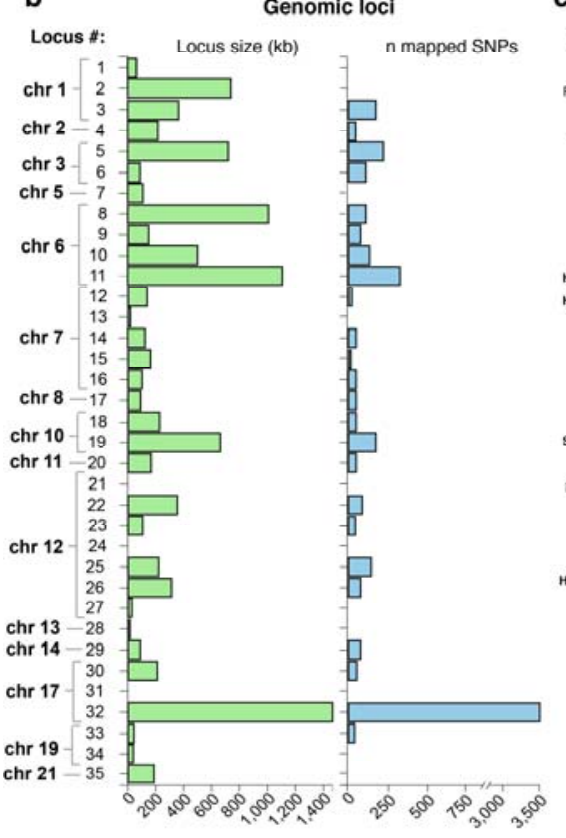

Category:

Partitioned heritabilty
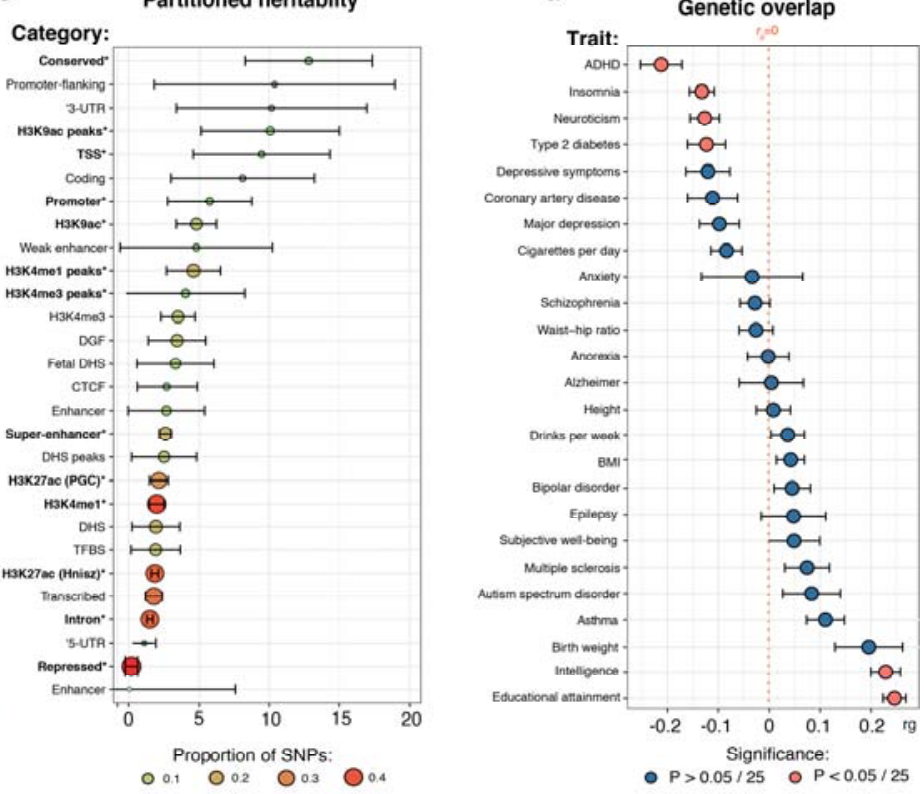

Figure 1. Genome-wide meta-analysis and follow-up analysis of brain volume (BV) in UK Biobank, ENIGMA-CHARGE and Early Growth Genetics consortium. (a) Manhattan plot of the GWAS metaanalysis of BV in 54,407 individuals showing the genomic position of each SNP on the $x$-axis and the metaanalysis negative $\log _{10}$-transformed $P$-value on the $y$-axis. Independent lead SNPs of each locus are annotated by a diamond. The horizontal red line indicates the genome-wide significance threshold that corresponds to a $P$ value of $5 \times 10^{-8}$, while the horizontal blue indicates the suggestive threshold of $1 \times 10^{-5}$. (b) Overview of the genomic loci sizes and number of SNPs mapped by each locus. (c) Partitioning of the SNP heritability of the BV GWAS meta-analysis by binary SNP annotations using LD Score regression. Enrichment was calculated by dividing the partial heritability of a category by the proportion of SNPs in that category. Significant enrichments, corrected for the number of categories tested $(P<0.05 / 28)$, are highlighted in bold and with an asterisk. (d) Genetic correlations with previous traits estimated using LD Score regression. Red dots denote significant genetic correlation after Bonferroni correction $(P<0.05 / 25)$. 
a

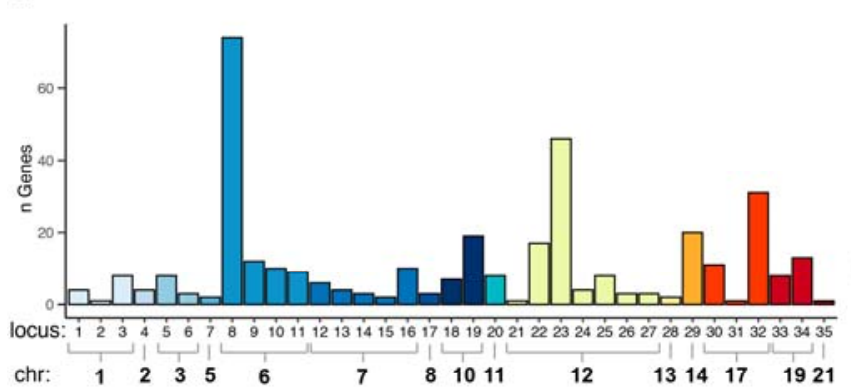

b

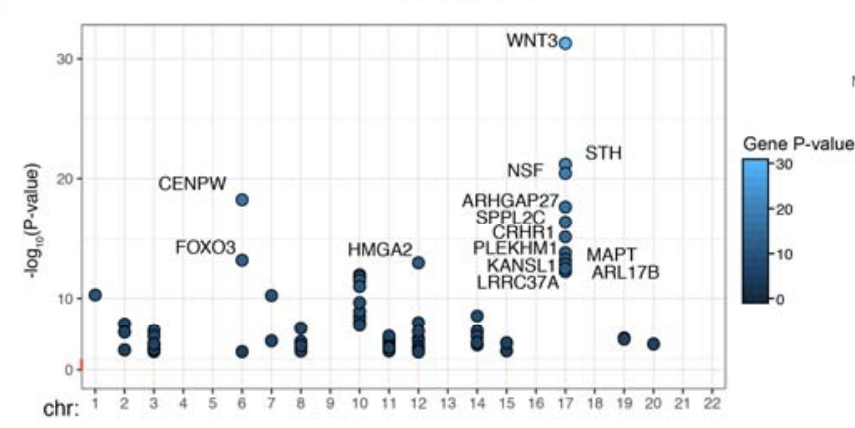

c
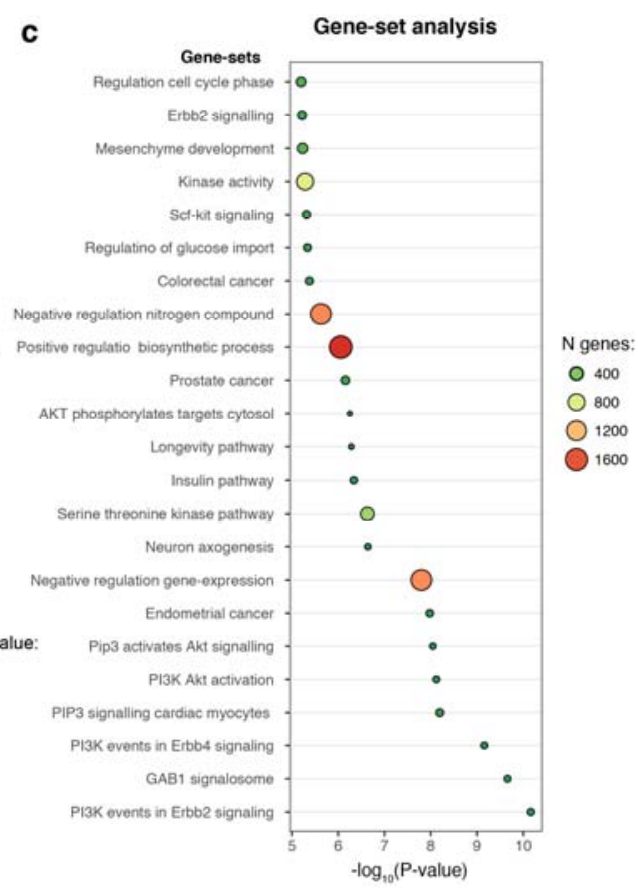

Figure 2. Gene analyses based on the GWAS meta-analysis of BV. (a) Number of genes that were mapped by FUMA to each locus. Colors indicate distinct chromosome numbers (b) Significant genes in the gene-based association test in MAGMA after Bonferroni correction $(P<0.05 / 18,161)$. The top 15 most significant genes are annotated with the corresponding gene symbols. (c) Significant MsigDB gene-sets after Bonferroni correction $\left(P<6.36 \times 10^{-6}(=0.05 /(7,246+53+565))\right.$ showing the negative $\log _{10}$-transformed $P$-value on the $x$-axis; size and colors of the circles represent the size of the gene set. 

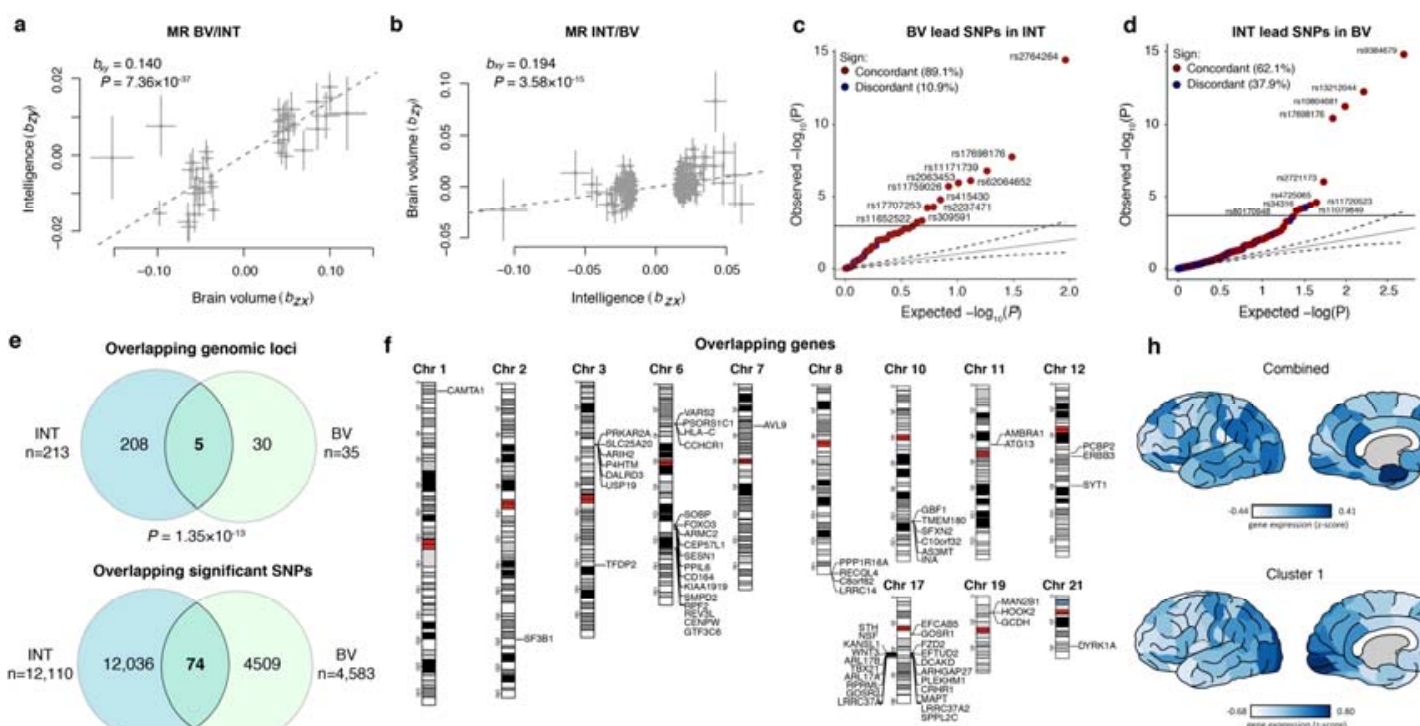

h
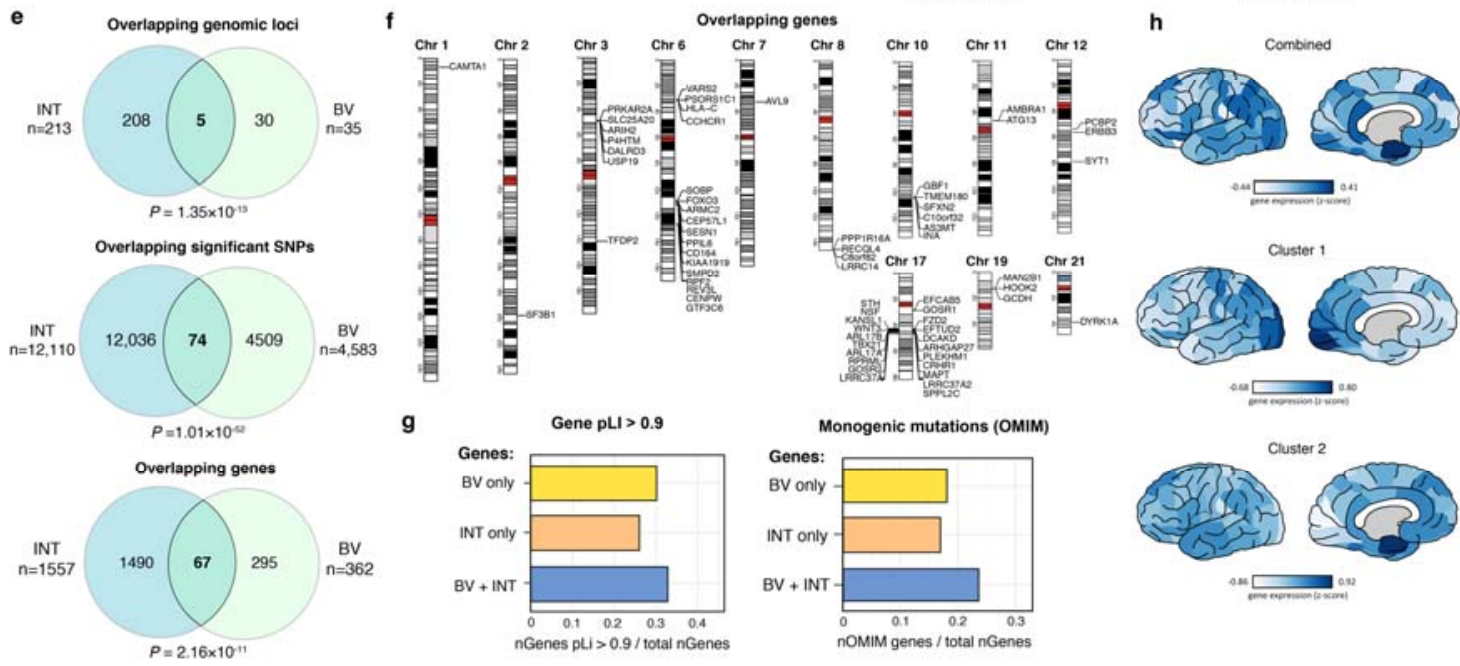

Figure 3. Analyses of the genetic overlap between BV and intelligence. (a) Mendelian Randomization analysis, showing the effects and standard errors of each SNP of BV $\left(b_{z y}\right)$ on intelligence $\left(b_{z x}\right)$ and the diagonal line showing the estimated causal effect $\left(b_{x y}\right)$ of BV on intelligence. (b) Similar to a) but of intelligence on BV. (c) Q-Q plot showing the observed and expected $P$-value distribution of 46 lead SNPs of the BV meta-analysis in intelligence and (d) 243 intelligence lead SNPs in BV. The colors denote the concordance (/discordance) of effects between the two traits. (e) Venn diagrams showing the number of overlapping loci, significant SNPs and genes implicated in BV and intelligence by FUMA and MAGMA. (f) Karyogram showing the genomic position of genes that were implicated in the GWAS of both BV and intelligence. (g) Comparison of the pLI score (representing tolerance to loss-of-function variants) and fraction of genes involved in developmental disorders between BV-only genes, intelligence-only genes (INT), and genes associated to bot traits (BV+INT). (h) Cortical gene expression patterns (colors indicate rank scores) across 57 cortical areas of the 67 genes that overlap between BV and intelligence, and the two clusters. 


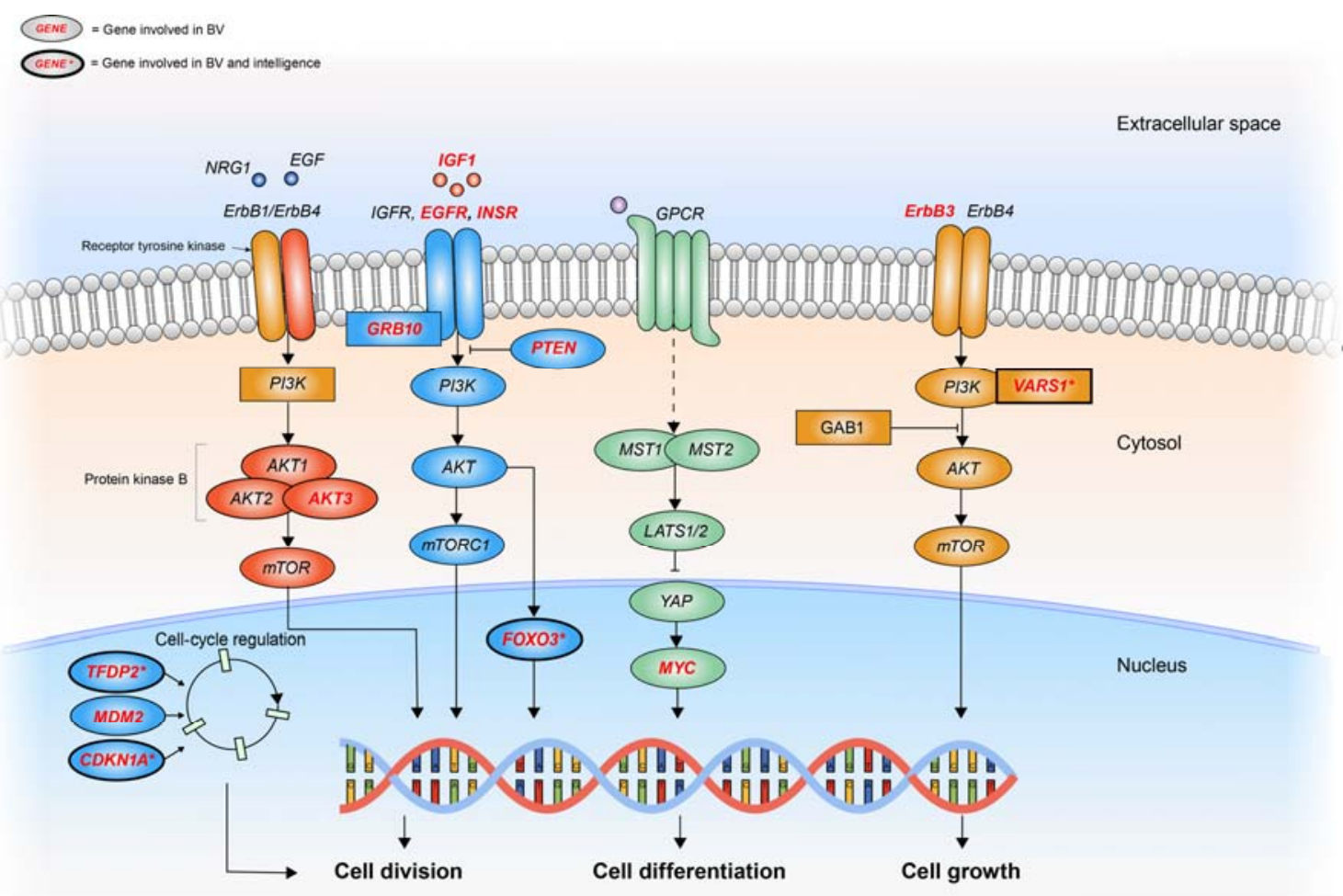

274 Fig 4. Schematic representation of cellular signaling pathways involved in BV. Overview of cellular signaling pathways and implicated genes within these pathways that were mapped by the GWAS metaanalysis of brain volume (BV) or overlapped with the genes that were mapped by the GWAS of intelligence. Genes in highlighted in red were involved in the meta-analysis of BV, whereas genes highlighted in red in a thick-outlined oval denoted by an asterisk were observed in both the BV and intelligence GWAS. 


\section{ONLINE METHODS}

283 Samples \& phenotypes

284 UK Biobank - Total brain volume

285 The UK Biobank (UKB) constitutes a large data set, combining a wide range of phenotypes with

286 genetic and imaging information. Here, we used processed data of a subset of $\mathrm{N}=21,407$

287 individuals who underwent a magnetic resonance imaging (MRI) procedure: data were released

288 in the third quarter of 2018. After filtering on quality of the imaging results, relatedness,

289 European ancestry and the availability of relevant covariates (discussed in more detail below),

290 we arrived at a final sample size of $\mathrm{N}=17,062$. The total brain volume (TBV) phenotype was

291 approximated as follows: TBV $=$ white matter volume + grey matter volume + cerebrospinal

292 fluid volume. The UKB obtained ethical approval from the National Research Ethics Service

293 Committee North West-Haydock (reference 11/NW/0382), and all study procedures were

294 performed in accordance with the World Medical Association for medical research. The current study was conducted under UKB application number 16406.

\section{ENIGMA-CHARGE collaboration - Intracranial volume}

298 We used GWAS summary statistics on intracranial volume obtained in a collaboration between

299 the Cohorts for Heart and Aging Research in Genomic Epidemiology (CHARGE) and Enhancing

300 NeuroImaging Genetics through Meta-Analysis (ENIGMA) ${ }^{35}$. ICV was calculated from brain MRI

301 data collected in 46 individual cohorts. Given the strong correlation between brain volume and

302 height ( $r=0.55$ in UK Biobank) we considered height an important covariate to include in GWAS

303 on volumetric brain measures. Therefore, we used mtCOJO to correct for height, by conditioning

304 on height using UKB-derived sumstats (this procedure is described in more detail below). Aside

305 from the full summary statistics $(\mathrm{N}=26,577)$, we also obtained summary statistics for a subset of

306 individuals for who 'height' was available as a covariate $(\mathrm{N}=21,875)$. Details on the individual

307 cohorts included in the ENIGMA-CHARGE collaboration are described elsewhere ${ }^{35}$. All 
308 participants were of European descent and provided informed consent. All studies included in

309 the ENIGMA-CHARGE collaboration were approved by their local institutional review board or

310 local ethics committee. Details on genotyping, imputation and analysis procedures can be found

311 in the original publication ${ }^{35}$.

313 EGG consortium

314 In contrast to the two samples discussed above, the GWAS summary statistics obtained from the

315 Early Growth Genetics (EGG) consortium are based on genetic analysis of an indirect measure of

316 brain volume, i.e., head circumference in infancy. These summary statistics were generated by

317 meta-analyzing seven population-based European studies, resulting in a total sample size of

$318 \mathrm{~N}=10,678$. All studies included in the meta-analysis were approved by the local ethics

319 committees, and informed (or parental) consent was obtained from all participants. Data

320 collection, genotyping, imputation and analysis procedures were described in detail

321 elsewhere ${ }^{36,37}$. Since not all individual studies that were included in the EGG consortium

322 corrected for height, we first conditioned the summary statistics on height using a procedure

323 similar to that described for the ENIGMA-CHARGE data.

\section{Genotyping, imputation \& quality control}

326 UK Biobank

327 The genotype data that we used was released by the UKB in March 2018, and concerns an

328 updated version of data released earlier (July 2017). Details on the collection and processing of

329 the genotype data are described elsewhere ${ }^{3}$. To summarize, the UKB genotyped in total 489,212

330 individuals on two custom made SNP arrays (UK BiLEVE Axiom ${ }^{\mathrm{TM}}$ array covering 807,411

331 markers $(\mathrm{n}=49,950)$ and UK Biobank ${ }^{\mathrm{TM}}$ Axiom array covering 825,927 markers $(\mathrm{n}=438,427)$,

332 both by Affymetrix). The genotype arrays shared 95\% of marker content. Quality control

333 executed by the UKB team resulted in a total of 488,377 individuals and 805,426 unique

334 markers in the released data. In the version of the data used for the current study (release 
335 March 2018), genotypes were phased and imputed by the UKB team to a combined reference

336 panel of the Haplotype Reference Consortium and the UK10K. Finally, imputed and quality-

337 controlled genotype data was available for 487,422 individuals and 92,693,895 genetic variants.

338 Prior to analysis, imputed variants were converted to hard call using a certainty threshold of

339 0.9. In our own quality control procedure, we excluded SNPs with a low imputation score (INFO

340 score $<0.9)$, low minor allele frequency $(\mathrm{MAF}<0.005)$ and high missingness $(>0.05)$.

341 Furthermore, indels positioned in the same chromosomal location were excluded. This resulted

342 in a total of 9,203,453 SNPs used for downstream analysis.

343 To prevent population stratification from biasing the results, we only included individuals from

344 European descent in our analyses. To this end, we projected principal components (PC's) from

345 the 1000 Genomes reference populations ${ }^{38}$ onto the called genotypes available in the UKB data.

346 Participants for whom the projected principal component score was closest (using the

347 Mahalanobis distance) to the average score of the European 1000 Genomes sample ${ }^{39}$ were

348 considered to be of European descent. Participants having a Mahalanobis distance $>6$ S.D. were

349 excluded from further analysis. In an additional quality control step, we excluded participants

350 that 1) had withdrawn their consent, 2) were related according to the UKB team (i.e., subjects

351 with most inferred relatives, $3^{\text {rd }}$ degree or closer, were removed until no related subjects were

352 present), 3) reported a gender that did not match their genetic gender, or 4) showed sex-

353 chromosome aneuploidy. After filtering availability of imaging data, and MRI scan quality,

35417,062 individuals remained for analysis.

\section{GWAS of total brain volume in UKB}

357 The genome-wide association analyses (GWAS) of total brain volume (BV) in the UKB data was

358 conducted in PLINK ${ }^{40,41}$, using a linear regression model with additive allelic effects. In order to 359 correct for potential subtle population stratification effects, we included 10 genetic PC's as 360 covariates. Genetic PC's were computed using FlashPC2 ${ }^{42}$ in the QC'ed subset of unrelated

361 European subjects, retaining only independent $\left(r^{2}<0.1\right)$, relatively common $(\mathrm{MAF}>0.01)$ and 
362 genotyped or very high imputation quality (INFO=1) SNPs ( $n=145,432$ markers). Additional

363 covariates included in the analysis were: age, sex, genotype array, assessment center, standing

364 height, and the Townsend deprivation index.

\section{Conditional GWAS (mtCOJo)}

367 We used multi-trait-based conditional \& joint analysis using GWAS summary data (mtCOJO see URLs) to conduct conditional GWAS analyses of our traits of interest. The mtCOJO method is integrated in the GCTA ${ }^{43}$ software, and requires summary-level GWAS data to perform a GWAS analysis of phenotype A, conditioned on phenotype B. In the current study, we performed two conditional GWASs, aiming to correct for height in two samples measuring ICV (ENIGMACHARGE) and head circumference (EGG), respectively. First, we ran a conditional GWAS for ICV in the full ENIGMA-CHARGE sample, conditioning on UKB-derived summary statistics for standing height. Second, we ran a similar conditional analysis for head circumference in the EGG sample, again conditioning on UKB-derived summary statistics for standing height. Results from the conditional GWASs were subsequently included in the main GWAS meta-analysis of brain volume described in the following section.

\section{GWAS meta-analysis of brain volume}

380 Before carrying out the meta-analysis of brain volume (BV), we performed additional filtering

381 and prepared the summary statistics for each of the individual cohorts. First of all, within the 382 ENIGMA-CHARGE data, SNPs that were available for $N<5,000$ were excluded. We then 383 performed similar filtering on the EGG data. Secondly, in the UKB data we identified some 384 instances where there were multiple SNPs on the same position, whilst having different alleles.

385 These SNPs were excluded from further analysis. Lastly, we aligned the allele coding of indels in 386 the ENIGMA-CHARGE data to match the coding in the UKB data. A number of indels that was not 387 present in the UKB data were excluded from analysis, since we used the UKB data as reference 388 data for downstream analyses. The summary statistics from the UKB, ENIGMA-CHARGE and the 
389 EGG consortium showed strong genetic correlations $\left(r_{g}\right.$ ranging between 0.71 and 1 ; see

390 Supplementary Table 2), supporting our choice for a meta-analytic approach.

391 Using a sample-size weighted $z$-score method in METAL ${ }^{10}$ we combined the GWAS on total brain

392 volume in the UKB data $(\mathrm{N}=17,062)$, the GWAS on intracranial volume (conditioned on height)

393 in the ENIGMA-CHARGE data $(\mathrm{N}=26,577)$, and the GWAS on head circumference (conditioned

394 on height) in the EGG data, resulting in a total sample size of N=54,407 (see Supplementary Fig.

395 1).

396 To validate our results, we conducted a second meta-analysis, using a stricter phenotype, and

397 only summary statistics from GWASs in which height was directly included as a covariate

398 (rather than conditioning on height using mtCOJO afterwards). In this stricter meta-analysis, we

399 thus combined the full summary statistics from the UKB $(N=17,062)$ with a subset of the

400 ENIGMA-CHARGE collaboration for which information on height had been available and

401 included as a covariate in the GWAS $(\mathrm{N}=21,875)$, resulting in a total sample size of $\mathrm{N}=38,937$.

402 The results of this validation meta-analysis are thus based on a purer BV phenotype (i.e.,

403 directly corrected for height and excluding head circumference as a proxy for BV). However,

404 given the high genetic correlations between the two meta-analyses for BV $\left(r_{\mathrm{g}}=.957, \mathrm{SE}=0.007\right)$

405 and the larger sample size, we expect statistical power to be greater in the primary meta-

406 analysis including all data. This larger meta-analysis was thus used for all subsequent analyses.

\section{Intelligence - GWAS summary statistics}

409 We used recently published GWAS meta-analysis summary statistics of intelligence ${ }^{2}$ to study the

410 genetic overlap with brain volume. Data collection procedures and methods are described in

411 detail elsewhere ${ }^{2}$. In comparing the results of $\mathrm{BV}$ and intelligence, we updated some of the 412 downstream analyses for intelligence (e.g., we used FUMA version 1.3.2 instead of 1.3.0, we 413 explored a slightly different collection of gene sets, and used GTEx data v7 instead of v6.1).

414 Therefore, gene-based findings presented in the current study may deviate slightly from those 
415 presented in the original study ${ }^{2}$. We applied Bonferroni correction for multiple testing to the

416 meta-analytic SNP P-values to identify to intelligence associated genetic variants.

\section{Genomic risk loci and functional annotation}

\section{SNP annotation}

420 We used FUMA ${ }^{12}$ (v1.3.2; see URLs) for the functional annotation of the BV meta-analysis 421 results. FUMA is an online platform that takes GWAS summary statistics as input, and 422 subsequently annotates, prioritizes, and visualizes the results.

423 Prior to defining genomic risk loci, FUMA identifies variants that are genome-wide significant $424\left(5 \times 10^{-8}\right)$, and independent $\left(r^{2}<0.6\right)$ as independent significant SNPS. In the next step, independent 425 significant SNPS that are independent from each other at $r^{2}<0.1$ are denoted lead SNPs. 426 Genotypes from the UKB were used as reference data to infer LD. Finally, FUMA characterizes 427 genomic risk loci by merging LD blocks that are located close to each other $(<250 \mathrm{~kb}$ apart $)$. 428 Thus, it is possible that one genomic risk locus contains multiple independent significant SNPs or 429 lead SNPs.

430 In order to obtain information on the functional consequences of SNPs on genes, FUMA 431 performs ANNOVAR $^{44}$ gene-based annotation using Ensembl genes (build 85) for all SNPs in LD $432\left(r^{2}>0.6\right)$ with one of the independent significant SNPS and having an association $P$-value lower 433 than $1 \times 10^{-5}$. Additionally, CADD scores ${ }^{45}$, Regulome DB scores ${ }^{46}$, and 15 -core chromatin 434 state $^{47,48}$ are annotated to SNPs by matching chromosome position, reference, and alternative 435 alleles. CADD scores can be used to prioritize genetic variants that are likely to be pathogenic 436 and/or deleterious (CADD scores $>12.37$ suggest a SNP is deleterious). The score is a single 437 measure combining various annotations, and has been shown to correlate with pathogenicity, 438 disease severity, and experimentally measured regulatory effects and complex trait

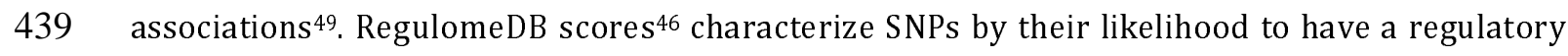
440 functions (with lower scores indicating higher probability of regulatory function). Scores range 441 from 7, meaning that there is no evidence of the variant having a regulatory function, to $1 \mathrm{a}$, 
442 meaning that a variant is likely to affect binding and is linked to expression of a gene target ${ }^{46}$.

443 Chromatin state was predicted by ChromHMM ${ }^{48}$ for 127 cell types, using 15 states to classify

444 and describe variants.

Gene mapping

447 Using FUMA ${ }^{12}$, all SNPs in genomic risk loci that were genome-wide significant $\left(P<5 \times 10^{-8}\right)$ or 448 were in LD $\left(r^{2}>0.6\right)$ with one of the independent significant SNPs, were mapped to genes. SNPs 449 could be annotated to a gene by either of three strategies. First, positional mapping maps SNPs 450 to protein coding genes based on physical proximity (i.e., within $10 \mathrm{~kb}$ window). Second, eQTL 451 mapping maps SNPs to genes whose expression is associated with allelic variation at the SNP 452 level. Information on eQTLs was derived from 3 publicly available data repositories; GTEx ${ }^{50}$ 453 (v7), the Blood eQTL browser ${ }^{51}$ and the BIOS QTL browser ${ }^{52}$. This strategy maps SNPs to genes 454 up to $1 \mathrm{Mb}$ apart (cis-eQTLs). We applied a false discovery rate (FDR) of $\leq 0.05$ to limit the 455 results to significant SNP-gene pairs. Third, SNPs were mapped to genes based on significant 456 chromatin interactions between promoter regions of genes $(250 \mathrm{bp}$ up- and $500 \mathrm{bp}$ 457 downstream of the transcription start site (TSS)) and a genomic region in a risk locus. In 458 contrast to eQTL mapping, and in the absence of a distance boundary, chromatin interaction 459 mapping may involve long-range interactions. The resolution of chromatin interactions was 460 defined as $40 \mathrm{~kb}$, and hence, interaction regions may comprise multiple genes. In order to 461 prioritize genes implicated by chromatin interaction mapping, information on predicted 462 enhancers and promoters in 111 tissue/cell types from the Roadmap Epigenomics Project ${ }^{53}$ was 463 integrated. We used FUMA to filter on chromatin interactions for which one interaction region 464 overlapped with predicted enhancers, and the other with predicted promoters 250 bp up- and $465500 \mathrm{bp}$ downstream of the TSS site of a gene. At the time of writing, FUMA contained Hi-C data 466 of 14 tissue types from the study of Schmitt et al. (2016) ${ }^{54}$. An FDR of $1 \times 10^{-5}$ was used to define 467 significant interactions. 
470 Genome-wide gene-based analysis (GWGAS) has the potential to identify genes associated to a 471 trait of interest despite the genetic signal of individual SNPs in or nearby the gene not reaching 472 genome-wide significance in SNP-based analyses. In contrast, a gene harboring a few strongly 473 associated SNP and many SNPs that show only very weak association may not be implicated by 474 gene-based analysis. So in addition to gene-mapping in FUMA, we conducted gene-based 475 analysis in MAGMA ${ }^{16}$ to assess the joint effect of all SNPs within all 19,427 protein-coding genes 476 included in the NCBI 37.3. MAGMA requires as input the $P$-values derived from SNP-based 477 analyses, in this case the BV meta-analysis results. All SNPs in our BV meta-analysis were annotated to genes, resulting in 18,161 and 18,162 genes that contained at least one SNP in the BV meta-analysis and the validation meta-analysis, respectively. Besides SNPs located within a 480 gene, we also included SNPs lying within $2 \mathrm{~kb}$ before and $1 \mathrm{~kb}$ after the TSS of the gene. We used

481 Entrez ID as the primary gene ID. MAGMA's gene-based analysis uses a multiple linear principal 482 component regression, where an $F$-test is used to compute the gene $P$-value. The model takes 483 linkage disequilibrium between SNPs into account. Genes were considered to be genome-wide 484 significantly associated if the $P$-value survived a Bonferroni correction for multiple testing $485\left(0.05 /\right.$ number of genes tested: $\left.P<2.75 \times 10^{-6}\right)$.

\section{Gene-set and tissue expression analysis}

\section{$488 \quad$ Functional gene-sets}

489 Gene-set analysis was performed using MAGMA ${ }^{16}$, testing 7,246 predefined gene sets in an 490 exploratory fashion. Selected gene sets included canonical pathways $(n=1,329)$ and gene 491 ontology (GO) gene sets $(n=5,917)$. All gene sets were obtained from the Molecular Signatures 492 Database (MSigDB, version 6.0; see URLs). For all gene-set analyses, competitive, rather than 493 self-contained, $P$-values are reported. Competitive gene-set analysis tests whether the joint 494 association of genes in a gene set with the phenotype of interest is stronger than that of a 495 randomly selected set of genes of the same size. This approach provides stronger evidence for 
496 association of the gene set compared to a self-contained test, where the joint association of

497 genes in a gene set with the phenotype is tested against the null hypothesis of no effect.

498

499 Gene-expression analysis

500 To assess whether genes associated to our traits of interest are disproportionately expressed in

501 certain tissue- and cell-types, we applied MAGMA's gene-expression analysis to investigate

502 associations with several gene expression profiles. First, we tested tissue gene-expression in 53

503 different tissue types obtained from the GTeX portal (v.7; see URLs), which include gene-

504 expression data from 13 brain tissue types ${ }^{20}$. Secondly, we tested gene-expression in 565

505 distinct adult mouse brain cell-types from Dropviz ${ }^{21}$. These data were collected through the use

506 of the Drop-seq technique ${ }^{55}$ by assessing RNA expression in 690,000 individual cells from 9

507 brain regions of the adult mice brain, which were subsequently grouped to 565

508 transcriptionally distinct groups of cell-types.

509 Gene-set and gene-expression analyses were Bonferroni corrected for the total number of gene-

510 sets, tissue types and single-cell types tested in MAGMA $\left(P<6.36 \times 10^{-6}\right.$

$511(=0.05 /(7,246+53+565))$.

512

\section{Conditional gene-set analyses}

514 In order to gain more insight in the genetic pathways associated to BV and intelligence, we

515 performed conditional gene-set analyses using MAGMA ${ }^{16}$. Conditional analyses were conducted

516 with the aim of identifying MsigDB gene sets that represent independent associations (i.e., in a

517 regression-based framework, we assessed the association between our trait of interest (e.g., BV)

518 and a gene set, conditional on another trait (e.g., intelligence)). Specifically, we determined

519 which gene set associations remain for BV when we condition on intelligence, and vice versa.

520 This approach provides information on whether gene sets are uniquely associated to BV or

521 intelligence, or rather, shared between both traits. For example, if the $P$-value of association

522 between a gene set and BV increases when conditioning on intelligence (i.e., less significant), 
523 then this suggests that the gene-set association is likely shared between both traits, while if the

$524 P$-value of a gene-set and BV is unaffected by conditioning on intelligence, then this implies that

525 the association is specific to BV, i.e., not shared with intelligence. In addition, we conducted

526 pairwise conditional gene-set analysis for all 23 gene-sets that were significantly associated to 527 BV.

SNP-based heritability and genetic correlations

530 We used linkage disequilibrium score regression (LDSC) ${ }^{11}$ to estimate the proportion of

531 phenotypic variance that can be explained by common SNPs, a statistic known as SNP-based

532 heritability, $h^{2}$ SNP. We used precomputed LD scores that were calculated using 1000 Genomes

533 European data (see URLs).

534 Genetic correlations $\left(r_{g}\right)$ between the signal from our BV meta-analysis, intelligence ${ }^{2}$, and 24

535 psychiatric, behavioral and lifestyle-related traits for which summary-level data were available,

536 were also calculated using $\operatorname{LDSC}^{9,11}$. Genetic correlations for which the $P$-value survived the

537 correction for multiple testing (Bonferroni-corrected $P<0.002(=0.05 / 25)$ ) were considered

538 significant.

\section{$540 \quad$ Partitioned heritability}

541 In order to determine whether some functional categories of the genome contribute more than 542 others to the SNP-heritability $\left(h^{2}{ }_{S N P}\right)$ of BV, we performed stratified LDSC ${ }^{56}$. Using this method, 543 we calculated whether any of the 28 specific genomic categories (see URLs) included in the 544 analysis was enriched for SNPs that contribute to $h^{2} S N P$. Enrichment here is defined as the 545 proportion of $h^{2}{ }_{S N P}$ in a given category divided by the proportion of SNPs in that category (e.g., if 546 enrichment in intronic regions is 4.75 , this indicates that this functional category is responsible 547 for a 4.75 -fold higher contribution to $h^{2}{ }_{S N P}$ compared to all tested SNPs). 
550 Mendelian randomization (MR) analysis was performed using Generalized summary-data-based

551 Mendelian randomization (GSMR ${ }^{30}$; see URLs). The main goal was to examine whether the

552 genetic correlation between brain volume and intelligence $\left(r_{g}=0.23\right)$ might be explained by

553 directional effects. Analyses were conducted using forward and reverse GSMR, testing for uni-

554 and bidirectional effects between all BV and intelligence.

555

Linking expression of overlapping genes for brain volume and intelligence to specific brain

\section{7 regions}

558 To identify brain regions that are associated with genes that are observed in BV and

559 intelligence, we first performed gene-mapping with MAGMA and FUMA based on the summary

560 statistics of the GWAS of each trait separately, after which we extracted the set of genes

561 implicated by any of the gene-mapping strategies in both traits.

562 Subsequently, we extracted the cortical gene-expression profile for each of the overlapping 563 genes from the Allen Human Brain Atlas $^{33}$ (AHBA), which describes gene-expression data per 564 gene across distinct cortical areas. An expression profile of the set was obtained by taking the 565 average expression across the 67 genes. We also performed clustering on the individual 566 correlation patterns for each of the expression profiles of each of the 67 genes separately, by 567 computing a $67 \times 67$ gene-to-gene correlation matrix, with the level of correlation between two 568 genes taken as the Pearson correlation coefficient between their cortical expression profiles.

569 This matrix was subdivided into clusters (also called modules) using Newman's modularity 570 algorithm ${ }^{57}$, maximizing for each cluster the intra-cluster cohesion and minimizing cohesion 571 between genes of different clusters. Per cluster, a cluster-level expression profile was computed 572 by calculating the average gene expression profile across all genes in the given cluster, resulting 573 in clusters of genes showing distinct brain maps. 
576 Aiming to determine whether genes associated to both BV and intelligence are over- (or under-)

577 expressed in specific brain regions, we performed interaction analysis as implemented in

578 MAGMA (v1.07b). This analysis tested whether the combined involvement of a gene set (here:

579 genes related to BV) and continuous gene properties (here: gene expression) is different from

580 their individual effects on intelligence. A positive interaction effect would suggest that, within

581 the set of BV-related genes, the relation between expression and gene-based Z-scores for

582 intelligence is stronger compared to other genes (i.e., all genes for which both expression data

583 from AHBA, as well as gene-based Z-scores for intelligence were available). This would indicate

584 that gene expression in that specific region plays a particular role in the genetic relation

585 between brain volume and intelligence.

586 An interaction analysis was conducted separately for gene expression in each of 57 brain

587 regions defined in the Allen Human Brain Atlas ${ }^{33}$ (AHBA). To control for potential variation in

588 general expression levels across genes, we conditioned on the marginal effects of the BV-related

589 gene set and average gene expression across all 57 regions, as well as for their interaction. The

590 set of BV-related genes consisted of all genes that were significant in MAGMA's gene-based

591 analysis and/or mapped from SNP-level results in FUMA. Interaction effects were deemed

592 significant if their $P$-value exceeded the Bonferroni-corrected threshold of $P<8.77 \times 10^{-4}$

$593(0.05 / 57)$

\section{Data availability}

596 Our policy is to make genome-wide summary statistics (sumstats) publicly available. Summary

597 statistics from our brain volume meta-analyses are available for download at the website of the

598 department of Complex Trait Genetics, CNCR (see URLs). The GWAS summary statistics from

599 the intelligence meta-analysis conducted by Savage et al. $(2018)^{2}$ are also available from this

600 website.

601 
603 The full GWAS summary statistics from the ENIGMA-CHARGE collaboration were downloaded

604 from http://enigma.ini.usc.edu/research/download-enigma-gwas-results/.

605 Data on infant head circumference has been contributed by EGG Consortium and was

606 downloaded from www.egg-consortium.org.

607 The Genotype-Tissue Expression (GTEx) Project was supported by the Common Fund of the

608 Office of the Director of the National Institutes of Health, and by NCI, NHGRI, NHLBI, NIDA,

609 NIMH, and NINDS. The data used for the analyses described in this manuscript were obtained

610 from the GTEx Portal (see URLs) on 06/12/2018.

611

612 This work was funded by The Netherlands Organization for Scientific Research (NWO Brain \&

613 Cognition 433-09-228, NWO MagW VIDI 452-12-014, NWO VICI 435-14-005 and 453-07-001,

614 645-000-003). P.R.J. was funded by the Sophia Foundation for Scientific Research (SSWO, grant

615 nr: S14-27).

616 Analyses were carried out on the Genetic Cluster Computer, which is financed by the

617 Netherlands Scientific Organization (NWO: 480-05-003), by the VU University, Amsterdam, the

618 Netherlands, and by the Dutch Brain Foundation, and is hosted by the Dutch National

619 Computing and Networking Services SurfSARA. This research has been conducted using the UK

620 Biobank Resource (application number 16406). We would like to thank the participants and

621 researchers who collected and contributed to the data.

\section{URLS}

624 Early Growth Genetics consortium: www.egg-consortium.org

625 ENIGMA: http://enigma.ini.usc.edu/research/download-enigma-gwas-results/

626 FUMA GWAS platform: http://fuma.ctglab.nl/

627 LD Score regression: https://github.com/bulik/ldsc

628 MAGMA: https://ctg.cncr.nl/software/magma

629 MsigDB gene-sets: http://software.broadinstitute.org/gsea/msigdb/index.jsp 
bioRxiv preprint doi: https://doi.org/10.1101/613489; this version posted April 19, 2019. The copyright holder for this preprint (which was not certified by peer review) is the author/funder, who has granted bioRxiv a license to display the preprint in perpetuity. It is made available under aCC-BY-NC-ND 4.0 International license.

630 GTEx portal: https://www.gtexportal.org/home/

631 Mendelian randomization - GSMR: http://cnsgenomics.com/software/gsmr/

632 Multi-trait-based conditional \& joint analysis using GWAS summary data (mtCOJO):

633 http://cnsgenomics.com/software/gcta/\#mtCOJO

634 COLOC R package: https://cran.r-project.org/web/packages/coloc/coloc.pdf

635 Allen Human Brain Atlas (AHBA): http://human.brain-map.org/

636 GeneCards: https://www.genecards.org/

637

638 


\section{References}

640 1. Posthuma, D. et al. The association between brain volume and intelligence is of genetic origin.

$641 \quad$ Nat. Neurosci. 5, 83 (2002).

642 2. Savage, J. E. et al. Genome-wide association meta-analysis in 269,867 individuals identifies

643 new genetic and functional links to intelligence. Nat. Genet. 1 (2018).

644 3. Bycroft, C. et al. Genome-wide genetic data on 500,000 UK Biobank participants. bioRxiv $645 \quad$ (2017).

646 4. Adams, H. H. H. et al. Novel genetic loci underlying human intracranial volume identified 647 through genome-wide association. Nat. Neurosci. 19, 1569 (2016).

648 5. Taal, H. R. et al. Common variants at 12q15 and 12q24 are associated with infant head $649 \quad$ circumference. Nat. Genet. 44, 532 (2012).

650 6. Hshieh, T. T. et al. Head circumference as a useful surrogate for intracranial volume in older adults. Int Psychogeriatr. 28, 157-162 (2017).

652 7. Martini, M., Klausing, A., Lüchters, G., Heim, N. \& Messing-Jünger, M. Head circumference $653-$ a useful single parameter for skull volume development in cranial growth analysis $\square$ ? Head $654 \quad$ Face Med. 14, 1-8 (2018).

655 8. Wolf, H., Kruggel, F., Hensel, A., Wahlund, L. \& Arendt, T. The relationship between head size and intracranial volume in elderly subjects. Brain Res. 973, 74-80 (2003).

657 9. Bulik-Sullivan, B. K. et al. An atlas of genetic correlations across human diseases and traits. Nat. Genet. 47, 1-9 (2015).

659 10. Willer, C. J., Li, Y., Abecasis, G. R. \& Overall, P. METAL: fast and efficient meta-analysis of genomewide association scans. Bioinformatics 26, 2190-2191 (2010).

661 11. Bulik-Sullivan, B. K. et al. LD Score regression distinguishes confounding from polygenicity 662 in genome-wide association studies. Nat. Genet. 47, 291-295 (2015).

663 12. Watanabe, K., Taskesen, E., Bochoven, A. van \& Posthuma, D. FUMA: Functional mapping 664 and annotation of genetic associations. Nat. Commun. 8, (2017).

665 13. Müller, S. A., Scilabra, S. D. \& Lichtenthaler, S. F. Proteomic substrate identification for 666 membrane proteases in the brain. Front. Mol. Neurosci. 9, 96 (2016). 
667 14. de Leeuw, C. A., Mooij, J. M., Heskes, T. \& Posthuma, D. MAGMA: Generalized Gene-Set

668 Analysis of GWAS Data. PLOS Comput. Biol. 11, e1004219 (2015).

669 15. McKusick, V. A. Mendelian Inheritance in Man and its online version, OMIM. Am. J. Hum.

$670 \quad$ Genet. 80, 588-604 (2007).

671 16. de Leeuw, C. A., Mooij, J. M., Heskes, T. \& Posthuma, D. MAGMA: Generalized gene-set analysis of GWAS data. PLoS Comput. Biol. 11, 1-19 (2015).

673 17. Tschopp, O. et al. Essential role of protein kinase $\mathrm{B} \gamma(\mathrm{PKB} \gamma / \mathrm{Akt} 3)$ in postnatal brain

674 development but not in glucose homeostasis. Development 132, 2943-2954 (2005).

675 18. Lee, J. H. et al. De novo somatic mutations in components of the PI3K-AKT3-mTOR pathway cause hemimegalencephaly. Nat. Genet. 44, 941 (2012).

677 19. Poduri, A. et al. Somatic activation of AKT3 causes hemispheric developmental brain 678 malformations. Neuron 74, 41-48 (2012).

679 20. Lonsdale, J. et al. The Genotype-Tissue Expression (GTEx) project. Nature Genetics 45, 580$680 \quad 585(2013)$.

681 21. Saunders, A. et al. Molecular diversity and specializations among the cells of the adult mouse $682 \quad$ brain. Cell 174, 1015-1030 (2018).

683 22. Nishiyama, A., Komitova, M., Suzuki, R. \& Zhu, X. Polydendrocytes (NG2 cells):

684 multifunctional cells with lineage plasticity. Nat. Rev. Neurosci. 10, 9 (2009).

685 23. Pfeiffer, S. E., Warrington, A. E. \& Bansal, R. The oligodendrocyte and its many cellular $686 \quad$ processes. Trends Cell Biol. 3, 191-197 (1993).

687 24. van der Schot, A. C. et al. Influence of genes and environment on brain volumes in twin pairs 688 concordant and discordant for bipolar disorder. Arch. Gen. Psychiatry 66, 142-151 (2009).

689 25. Posthuma, D. et al. Genetic correlations between brain volumes and the WAIS-III dimensions 690 of verbal comprehension, working memory, perceptual organization, and processing speed. 691 Twin Res. Hum. Genet. 6, 131-139 (2003).

692 26. Posthuma, D. et al. Multivariate genetic analysis of brain structure in an extended twin design. 693 Behav. Genet. 30, 311-319 (2000).

694 27. Okbay, A. et al. Genome-wide association study identifies 74 loci associated with educational 
695 attainment. Nature 533, 539 (2016).

696 28. Zhao, B. et al. GWAS of 19,629 individuals identifies novel genetic variants for regional brain

697 volumes and refines their genetic co-architecture with cognitive and mental health traits.

$698 \quad$ bioRxiv 586339, (2019).

699 29. Roth, G. \& Dicke, U. Evolution of the brain and intelligence. Trends Cogn. Sci. 9, 250-257

$700 \quad(2005)$.

701 30. Zhu, Z. et al. Causal associations between risk factors and common diseases inferred from

702 GWAS summary data. Nat. Commun. 9, 224 (2018).

703 31. Davies, N. M., Holmes, M. V. \& Davey Smith, G. Reading Mendelian randomisation studies:

704 A guide, glossary, and checklist for clinicians. BMJ 362, (2018).

705 32. Rebhan, M., Chalifa-Caspi, V., Prilusky, J. \& Lancet, D. GeneCards: a novel functional

706 genomics compendium with automated data mining and query reformulation support.

707 Bioinformatics 14, 656-664 (1998).

708 33. Sunkin, S. M. et al. Allen Brain Atlas: an integrated spatio-temporal portal for exploring the

709 central nervous system. Nucleic Acids Res. 41, D996-D1008 (2012).

710 34. Wallace, C. et al. Statistical colocalization of monocyte gene expression and genetic risk

711 variants for type 1 diabetes. Hum. Mol. Genet. 21, 2815-2824 (2012).

712 35. Adams, H. H. H. et al. Novel genetic loci underlying human intracranial volume identified

713 through genome-wide association. Nat. Neurosci. 19, 1569-1582 (2016).

714 36. Rob Taal, H. et al. Common variants at $12 \mathrm{q} 15$ and $12 \mathrm{q} 24$ are associated with infant head

$715 \quad$ circumference. Nat. Genet. 44, 532-538 (2012).

716 37. Ikram, M. A. et al. Common variants at $6 \mathrm{q} 22$ and $17 \mathrm{q} 21$ are associated with intracranial

717 volume. Nat. Genet. 44, 539-544 (2012).

718 38. Auton, A. et al. A global reference for human genetic variation. Nature 526, 68-74 (2015).

719 39. Webb, B. T. et al. Molecular genetic influences on normative and problematic alcohol use in a

720 population-based sample of college students. Front. Genet. 8, 1-11 (2017).

721 40. Purcell, S. et al. PLINK: a tool set for whole-genome association and population-based linkage 722 analyses. Am. J. Hum. Genet. 81, 559-575 (2007). 
723 41. Chang, C. C. et al. Second-generation PLINK: rising to the challenge of larger and richer

724 datasets. Gigascience 4, 1 (2015).

725 42. Abraham, G. \& Inouye, M. Fast principal component analysis of large-scale genome-wide 726 data. PLoS One $\mathbf{9}, 1-5$ (2014).

727 43. Yang, J., Lee, S. H., Goddard, M. E. \& Visscher, P. M. GCTA: a tool for genome-wide complex trait analysis. Am. J. Hum. Genet. 88, 76-82 (2011).

44. Wang, K., Li, M. \& Hakonarson, H. ANNOVAR: functional annotation of genetic variants from high-throughput sequencing data. Nucleic Acids Res. 38, e164-e164 (2010).

45. Kircher, M. et al. A general framework for estimating the relative pathogenicity of human genetic variants. Nat. Genet. 46, 310-315 (2014).

46. Boyle, A. P. et al. Annotation of functional variation in personal genomes using RegulomeDB. Genome Res. 22, 1790-1797 (2012).

47. Roadmap Epigenomics Consortium et al. Integrative analysis of 111 reference human epigenomes. Nature 518, (2015).

48. Ernst, J. \& Kellis, M. ChromHMM: automating chromatin-state discovery and characterization. Nat. Methods 9, 215-216 (2012).

49. Kircher, M. et al. A general framework for estimating the relative pathogenicity of human genetic variants. Nat. Genet. 46, 310-315 (2014).

741 50. The GTEx Consortium. The Genotype-Tissue Expression (GTEx) pilot analysis: Multitissue gene regulation in humans. Science 348, 648-660 (2015).

743 51. Westra, H.-J. et al. Systematic identification of trans-eQTLs as putative drivers of known 744 disease associations. Nat. Genet. 45, 1238-1243 (2013).

745 52. Zhernakova, D. V et al. Identification of context-dependent expression quantitative trait loci in 746 whole blood. Nat Genet 49, 139-145 (2017).

747 53. Roadmap Epigenomics Consortium et al. Integrative analysis of 111 reference human $748 \quad$ epigenomes. Nature 518, 317-330 (2015).

749 54. Schmitt, A. D. et al. A compendium of chromatin contact maps reveals spatially active regions 750 in the human genome. Cell Rep. 17, 2042-2059 (2016). 
751 55. Macosko, E. Z. et al. Highly parallel genome-wide expression profiling of individual cells

$752 \quad$ using nanoliter droplets. Cell 161, 1202-1214 (2015).

753 56. Finucane, H. K. et al. Partitioning heritability by functional annotation using genome-wide

754 association summary statistics. Nat. Publ. Gr. 47, 1228-1235 (2015).

755 57. Newman, M. E. J. Modularity and community structure in networks. PNAS 103, 8577-8582

$756 \quad$ (2006).

757

758 
Supplementary Information includes:

760

761

762

763

764

765

766

767

768

769

770

771

772

773

774

775

776

777

778

779

780

\section{Supplementary Methods \\ 1.1 UK Biobank \\ 1.2 UK Biobank genotype data}

\section{Supplementary Results}

1. Results of GWAS meta-analysis of BV

1.1 UKB, ENIGMA-CHARGE \& EGG data

1.2 Functional annotation

1.3 Gene mapping results

1.4 Gene-set results

2. Validation GWAS meta-analysis

2.1 GWAS meta-analysis on strictly ICV in UKB and ENIGMA-CHARGE

2.2 Functional annotation

2.3 Gene mapping results

2.4 Gene-set results

2.5. Overlap between both meta-analyses

2.6. Gene-card summary of BV genes overlapping with intelligence

3. Supplementary Figures (1 to 13 )

4. Supplementary Tables (1 to 28 ) 